\title{
Um Estudo de Viabilidade para Avaliação de Atributos de Qualidade em uma Ferramenta Web de Submissão de Trabalhos Acadêmicos
}

\author{
Erik Aceiro Antonio \\ ${ }^{1}$ Escola Superior de Tecnologia e Educação de Rio Claro (ASSER) \\ Rio Claro - Rua 7, 1193 - Centro - CEP 13500-200 \\ sinfeasser.com.br
}

\begin{abstract}
Activities related to scientific research involves special issues on academic training - mainly when these activities are used as students-centered activities. This paper aims to show a feasibility study about a web tool that support submission review process associated to scientific review workflow. So, the following activities were performed - (i) collectiong metrics (KLoC) from each tool version; (ii) visualizing the code using the metrics $(i)$ projected through by CodeFlowers; and (iii) appling a feasibility study aiming to characterize the user-experience. In summary, we can evidence that the web tool evaluated has quality attributes, in particular, usability.
\end{abstract}

Resumo. Atividades relacionadas à pesquisa científica envolvem aspectos especiais na formação acadêmica - principalmente quando utilizadas como atividades centradas no estudante. Este artigo tem como objetivo apresentar um estudo de viabilidade sobre uma ferramenta web que suporta o workflow na revisão e submissão de trabalhos academicos associados ao fluxo de revisão científica. Assim, as seguintes atividades foram realizadas - (i) coletar métricas (KLoC) de cada versão da ferramenta; (ii) visualizar o código usando as métricas (i) projetadas via CodeFlowers; e (iii) aplicar um estudo de viabilidade visando caracterizar a experiência do usuário. Em resumo, pode-se verificar que a ferramenta possui atributos de qualidade, em especial, usabilidade.

\section{Introdução}

Atividades e disciplinas que envolvam Trabalhos de Conclusão de Curso (TCCs), Atividades Complementares (ACs) e Projetos de Extensão (PE) são essenciais na formação dos estudantes, uma vez que essas atividades favorecem, por exemplo, a colaboração e estimulam o pensamento crítico. Ressalta-se que, tais atividades devem estar em consonância com as diretrizes curriculares nacionais de cada curso de graduação em computação [MEC 2016]. Para favorecer o envolvimento e engajamento dos estudantes, novas iniciativas tem sido empregadas na direção do uso de tecnologias, ambientes e sistemas computacionais voltados especialmente para apoiar o ensino-aprendizagem [Oliveira et al. 2018]. Ferramentas TICs para a gestão de conteúdos, gerenciamento de submissões e revisão de artigos para eventos científicos é comum na área de computação o uso do JEMS (Journal and Event Management System). Contudo, ainda nota-se uma carência por relatos de experiências e estudos experimentais sobre ferramentas para a gestão do processo de 
VIII Congresso Brasileiro de Informática na Educação (CBIE 2019)

Anais do XXV Workshop de Informática na Escola (WIE 2019)

submissão de trabalhos acadêmicos, sobretudo, com relação à aspectos de qualidade e características.

Nesse contexto, este trabalho visa apresentar um relato de experiência envolvendo uma ferramenta web para apoiar o fluxo de trabalho relacionado com a submissão e revisão de trabalhos acadêmicos. Para isso, este trabalho está organizado da seguinte forma: nessa seção destacamos as motivações iniciais relacionadas com o trabalho e fundamentação teórica; em seguida na Seção 2 apresenta-se a metodologia para coletar e caracterizar indícios de interesse para planejar o estudo de viabilidade sobre a ferramenta; na Seção 3 apresenta-se uma descrição geral sobre a ferramenta Gnosis; na Seção 5 apresenta-se a sumarização do estudo de viabilidade e finalmente na Seção 6 a conclusão.

\section{Metodologia}

Seguindo as diretrizes de classificação de trabalhos acadêmicos proposta por [Gil 2002], este trabalho consiste em uma pesquisa científica aplicada e descritiva para a caracterização de uma ferramenta web. Com relação a abordagem, trata-se de uma pesquisa quantitativa e qualitativa onde por meio de um Estudo de Viabilidade procurou-se avaliar a existencia (ou não) de atributos de qualidade através da aplicação de um questionário para avaliação da usabilidade.

Desse modo, para a elaboração do estudo de viabilidade a estratégia apresentada na Figura 1 foi definida. Em (i) coletar métricas - é a primeira atividade realizada e consiste em coletar métricas de qualidade. Para isso, optou-se pela métrica $K \mathrm{LoC} / \mathrm{LoC}$ a partir das versões da ferramenta ( $v 1, v 2, v 3$ e $v 4$ ) via CLOC [Danial 2019]; (ii) visualizar código - é segunda atividade realizada e consiste em projetar as métricas obtidas pelo código-fonte ( $i$ )para visualização via CodeFlower [Zaninotto 2014]. Ressalta-se que, após a visualização do código foram determinados indícios e aspectos de qualidade que pudessem ser usados sobretudo na avaliação da ferramenta; finalmente em (iii) planejar estudo de viabilidade - envolve uma atividade para avaliar a ferramenta a partir do ponto de vista de estudantes de graduação. Ressalta-se que o planejamento do estudo de viabilidade envolveu as diretrizes propostas por [Wohlin 2012].

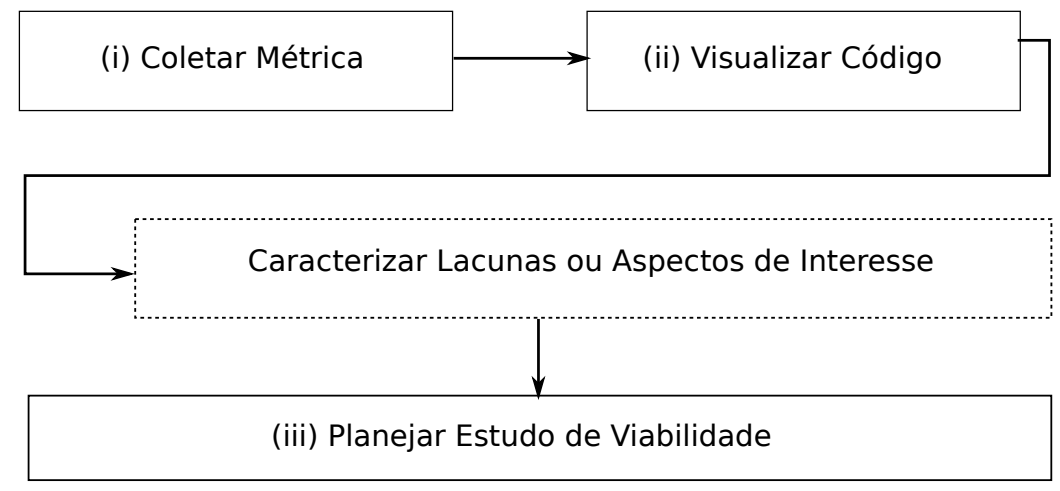

Figura 1. Metodologia elaborada para (i) Coletar, (ii) Visualizar e (iii) Planejar o Estudo de Viabilidade.

Considerando-se a importância na avaliação e mensuração de projetos na área de Engenharia de Software em geral [Pressman 2009][Danial 2019] destaca-se que a métrica 
VIII Congresso Brasileiro de Informática na Educação (CBIE 2019)

Anais do XXV Workshop de Informática na Escola (WIE 2019)

$K L o C$ (Line of Code) foi estabelecida neste trabalho como apresentado na Equação 1, onde $M$ é a quantidade de linhas de código existente e $T$ o total de linhas por arquivo. Essa métrica foi utilizada na atividade (i) e (ii) (Figura 1).

$$
K L o C=\sum_{l=1}^{M}\left[T_{\text {linhas }}-\left(T_{\text {linhas em branco }}-T_{\text {linhas de comentários }}\right)\right]
$$

$\mathrm{Na}$ atividade (iii)(Figura 1) foram elaborados os artefatos usados no estudo. Nesse caso, os seguintes itens foram organizados - termo de aceite, questionário e apresentação sobre a avaliação de usabilidade. Com relação ao artefato questionário, sete questões quantitativas $(Q 1-Q 7)$ e uma questão qualitativa $(Q 8)$ foram definidas. As questões $(Q 1-Q 7)$ foram definidas na escala de cinco-pontos de Likert, onde participantes avaliaram as questões em $1=$ ruim, $2=$ razoável, $3=$ bom, $4=$ muito bom e $5=$ excelente (vide Tabela 1). Salienta-se que a escala de Likert tem sido amplamente empregada em vários estudos, com destaque para aque-les envolvendo pesquisas de satisfação e usabilidade [Sauro 2009][Joost 2010]. Para esse estudo, os sujeitos selecionados foram onze estudantes $(n=11)$ do $2^{\circ}$ Período do Curso de Bacharelado em Sistemas de Informação. Ressalta-se também, que os estudantes em questão nunca tiveram contato prévio com a ferramenta.

Assim, visando o objetivo do estudo, a seguinte hipótese foi estabelecida.

$$
\begin{aligned}
& H_{0}: m_{Q}=\mu \text { i.e., não existem atributos de qualidade que permitam carac- } \\
& \text { terizar a usabilidade da ferramenta como satisfatória } \\
& H_{a}: m_{Q}>\mu
\end{aligned}
$$

onde $m_{Q}$ denota o escore em relação à média de $Q 1-Q 6$ para o Critério Excelente (CE) definido na Equação 2 e $\mu=50 \%$. Frequentemente, essa abordagem tem sido recomendada, uma vez que auxilia na investigação de conceitos menos conretos, como por exemplo, a satisfação dos participantes [Sullivan 2013].

$$
m_{Q}=\frac{\sum_{q=1}^{6} C E_{q}}{6}
$$

\section{Descrição da Ferramenta Gnosis}

Essa seção visa apresentar a ferramenta Gnosis $^{1}$. Periodicamente vinculado com eventos que ocorrem a cada semestre - Mostra Científica e Semana do Conhecimento - a ferramenta Gnosis é uma plataforma web que visa gerenciar trabalhos acadêmicos que são submetidos e avaliados por revisores e devem estar relacionados aos seguintes típos de trabalhos - palestras, seminários, mini-cursos e apresentações de trabalhos dos discentes e docentes [Antonio 2019b]. Destaca-se também que grande parte dos trabalhos apresentados, estão relacionados ao núcleo comum de disciplinas de Metodologia Científica, e

\footnotetext{
${ }^{1} \mathrm{O}$ nome Gnosis foi inspirado na etimologia do termo, que origina-se no Grego antigo e relaciona knowledge (conhecimento) e a busca pela verdade.
} 
VIII Congresso Brasileiro de Informática na Educação (CBIE 2019)

Anais do XXV Workshop de Informática na Escola (WIE 2019)

\begin{tabular}{l}
\hline Questão \\
\hline \hline Q1 - Qual a sua percepção com relação a facilidade de uso da ferra- \\
menta para realizar o login (acesso) em uma escala de 1(ruim) até 5(ex- \\
celente)? \\
\hline Q2 - Qual a sua percepção com relação a facilidade de uso da fer- \\
ramenta para a submissão de trabalhos acadêmicos em uma escala de \\
1(ruim) até 5(excelente)? \\
\hline Q3 - Durante a submissão de trabalhos acadêmicos (artigos, resumos \\
e mini-cursos) um ou mais feedbacks são apresentados na forma de di- \\
cas em balões. Você considera essa(s) informações relevantes para a \\
submissão de trabalhos em uma escala de 1(ruim) até 5(excelente)? \\
\hline Q4 - Com relação aos padrões de cores da ferramenta, você considera \\
que as cores estão padronizadas e proporcionam um agradável uso em \\
uma escala de 1(ruim) até 5(excelente)? \\
\hline Q5 - Com relação à disposição dos menus, botões e elementos visuais e \\
gráficos. Você considera que tais elementos proporcionam um amigável \\
uso da ferramenta em uma escala de 1(ruim) até 5(ótimo)? \\
\hline Q6 - Com relação ao conteúdo (texto) da ferramenta. Você considera \\
que o conteúdo é claro e proporciona um amigável entendimento em \\
uma escala de 1(ruim) até 5(excelente)? \\
\hline Q7 - Dentre as características - Simples, Complexo, Lento, Rápido e \\
Confuso - qual você considera como um indicativo de qualidade OU \\
ausência de qualidade da plataforma de eventos. \\
\hline Q8 - Questão aberta com críticas e sugestões sobre a ferramenta.
\end{tabular}

Tabela 1. Questionário para avaliação de usabilidade da ferramenta Gnosis

também dos respectivos Trabalhos de Conclusão de Curso (TCCs), Atividades Complementares (ACs) e Projetos de Extensão (PE).

A Tabela 2 destaca as atividades desenvolvidas - desde 2016 na ferramenta. Podese perceber que a ferramenta tem evoluído gradativamente, por exemplo, onde novas funcionalides relacionadas com a usabilidade foram especialmente incluídas. Tais atividades tem sido incluídas através do método de desenvolvimento Ágil guiado por Kanban [Pressman 2009]. A Figura 2 ilustra as principais da ferramenta.

Em geral, as principais funcionalidades podem ser observadas conforme os itens destacados na Figura 2 (a)-(d). Na Figura 2 (a) destaca-se a página principal de welcome, e como exemplo, um destaque sobre o conteúdo de referência da mostra; na Figura 2 (b) está sendo apresentado como é realizado o acesso ao ambiente, no caso, o acesso ocorre via a login com credencias já existentes ou a partir de um novo cadastro; na Figura 2 (c) pode-se visualizar a tela principal com o main-dashboard indicando as principais ações que o perfil de estudante permite acessar - nesse caso, submeter um resumo, verificar um resumo submetido (visualizar, remover e consultar) e geração de certificados digitalizados; e finalmente, na Figura 2 (d) está sendo ilustrada, como exemplo, os principais campos usados para submeter um resumo. 
VIII Congresso Brasileiro de Informática na Educação (CBIE 2019)

Anais do XXV Workshop de Informática na Escola (WIE 2019)

\begin{tabular}{|c|c|c|}
\hline Ano & Versão & Funcionalidade \\
\hline \multirow{5}{*}{2018} & $\overline{v e 4.0 .2}$ & $\begin{array}{l}\text { Geração de certificados digitalizados (automaticamente) } \\
\text { para alunos inscritos em } 2018 \text { e } 2017\end{array}$ \\
\hline & \multirow[t]{2}{*}{$v 4.0 .1$} & $\begin{array}{l}\text { Suporte a manuais e vídeos para auxiliar o workflow de sub- } \\
\text { missão }\end{array}$ \\
\hline & & Melhoria em usabilidade geral da ferramenta \\
\hline & \multirow[t]{2}{*}{$v 4.0 .0$} & $\begin{array}{l}\text { Versão estável para produção (PRD) liberada em } 17 \text { de nov. } \\
\text { de } 2018\end{array}$ \\
\hline & & $\begin{array}{l}\text { Refatoração e melhorias em funcionalidades para adicionar, } \\
\text { consultar orientadores e usabilidade geral - responsividade }\end{array}$ \\
\hline \multirow[t]{3}{*}{2017} & \multirow[t]{3}{*}{$v 3.0 .0$} & $\begin{array}{l}\text { Versão estável para produção (PRD) liberada em } 13 \text { de nov. } \\
\text { de } 2017\end{array}$ \\
\hline & & $\begin{array}{l}\text { Adicionada melhoria para responsividade e usabilidade no } \\
\text { workflow de submissão de resumos }\end{array}$ \\
\hline & & $\begin{array}{l}\text { Adicionado perfil para controle acadêmico - professores e } \\
\text { secretaria }\end{array}$ \\
\hline \multirow[t]{3}{*}{2016} & \multirow[t]{2}{*}{$v 2.0 .0$} & $\begin{array}{l}\text { Versão estável para produção (PRD) liberada em } 11 \text { de ou- } \\
\text { tubro de } 2016\end{array}$ \\
\hline & & $\begin{array}{l}\text { Adicionada melhoria para o Backend - Repository e PDOs } \\
\text { - suporte responsivo para submissão de Resumos }\end{array}$ \\
\hline & $v 1.0 .0$ & $\begin{array}{l}\text { Versão estável para produção (PRD) liberada em } 6 \text { de maio } \\
\text { de } 2016\end{array}$ \\
\hline
\end{tabular}

Tabela 2. Funcionalidades desenvolvidas na ferramenta Gnosis

Ressalta-se que a Figura 2 (a)-(d) ilustra apenas o perfil usado para submissão de trabalhos - resumos. A ferramenta também possui, o perfil de avaliador, onde avaliadores credenciados realizam a avaliação dos trabalhos através de chave de acesso por área de domínio.

\section{Caracterização da Ferramenta Gnosis}

Essa sessão apresenta a caracterização da ferramenta via métrica $K L o C$ (Figura 1 (atividade $i$ )) e da projeção do código via CodeFlower (Figura 1 (atividade ii)). Essa caracterização tem como objetivo fornecer subsídios para a posterior formulação do estudo de viabilidade. Na Figura 3 verifica-se a evolução da ferramenta a partir das métricas de qualidade coletadas $K L o C$ projetadas na visualização de código nas versões - $v 1, v 2$, $v 3$ e $v 4$ (Figura 3 (a)-(d)).

Essa taxa de crescimento de código apresentada na Figura 4, também ocorre notadamente na Figura 3 (d), onde pode-se verificar um maior número de nós conectados (acoplamento) em relação a Figura 3 (a). Nesse caso, deixando em evidência um maior número de nós em destaque. A partir da correlação e inferencia entre as Figuras 3 (a)(d) e Figura 4 pode-se notar que a ferramenta Gnosis, vem sendo incorporada de novas funcionalidades sobretudo vinculadas com funcionalidades centradas no usuário, como por exemplo, usabilidade. Por exemplo, na Figura 4 nota-se um aumento na quantidade de instruções de código relacionados com as linguagens PHP, HTML, CSS e JavaScript $(J S)$. 
VIII Congresso Brasileiro de Informática na Educação (CBIE 2019)

Anais do XXV Workshop de Informática na Escola (WIE 2019)

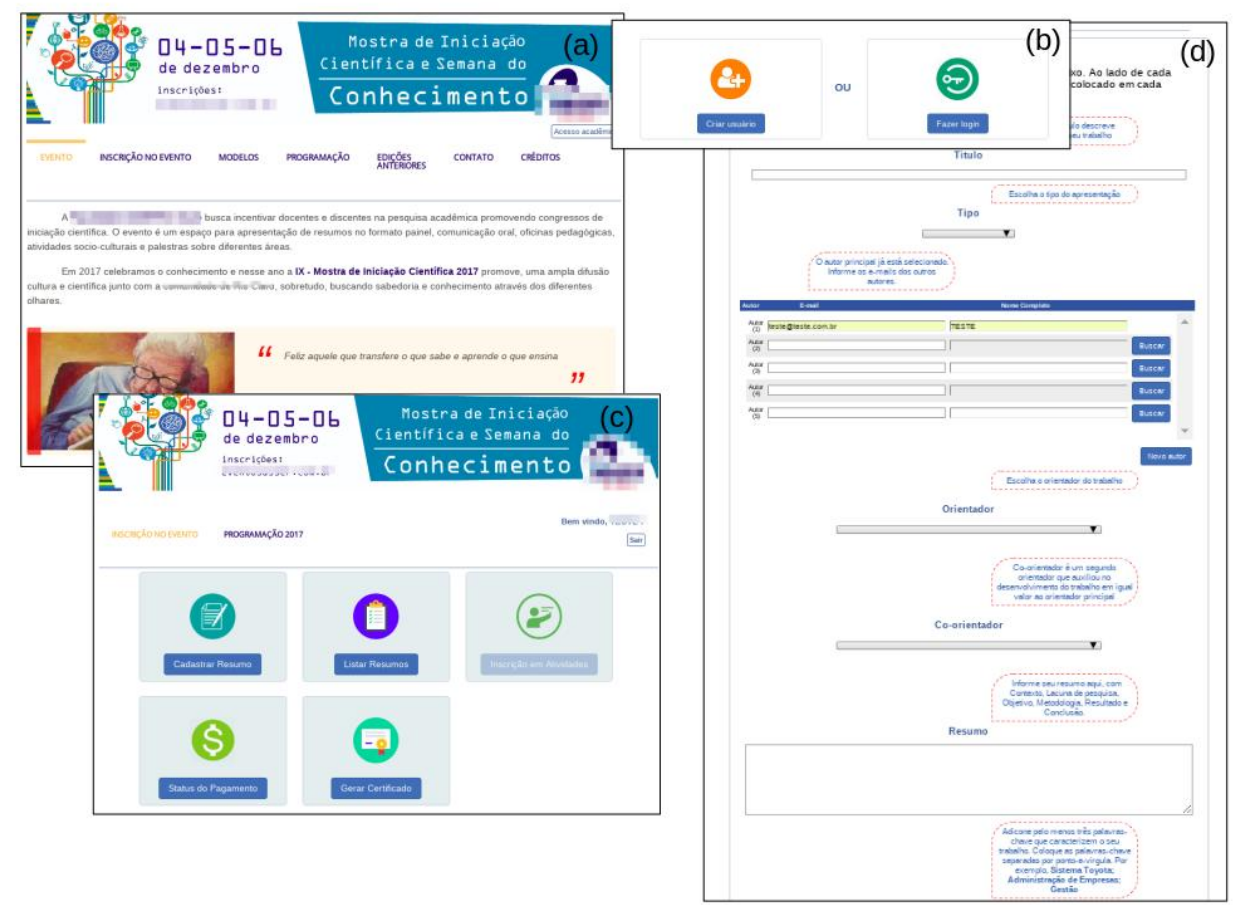

Figura 2. Principais telas da ferramenta da ferramenta (Gnosis) para avaliaçãorevisão de trabalhos acadêmicos

Nesse contexto, considerando-se $(a)$ a evolução que a ferramenta vem apresentando nos últimos anos conforme pode-se inferir nas Figuras 3 (a)-(d) e Figura 4; e (b) considerando-se a adição de novas funcionalidades que impactam a experiência do usuário, um estudo de viabilidade foi proposto visando especialmente avaliar a ferramenta Gnosis sobre aspectos de usabilidade e experiência de usuário. A Sessão 5 apresenta os resultados encontrados na execução do estudo viabilidade.

\section{Sumarização - Estudo de Viabilidade}

Como exposto na Sessão 2 a atividade final (iii) (Figura 1) consiste em planejar, executar e sumarizar o estudo de viabilidade visando a avaliação da ferramenta. Nesse caso, essa sessão descreve os principais resultados encontrados (Figuras 5 (a)-(g)) e Tabela 1. O formulário de avaliação, consentimento, dados brutos dos partipantes e scripts em $\mathrm{R}$ usados podem ser encontrados no repositório do autor [Antonio 2019a].

As Figuras 5 (a)-(f) podem ser interpretadas atavés da inferência dos gráficos de barra relacionando-se a questão respondida versus a frequência de pontuação. Por exemplo, a Figura 5 (a) indica a opinião geral dos participantes com relação a questão $Q 1$ (vide Tabela 1), o que nesse caso sugere que a ferramenta possui facilidade para se realizar login sobre o ponto de vista dos participantes. Ressalta-se, que dos 11 participantes, 8 deles ponturam que a ferrementa é excelente; 2 ponturam que a ferramenta é muito boa; e 1 participante pontuou que a ferramenta é boa. No mesmo gráfico, nota-se que nenhum participante indicou que a ferramenta é ruim ou razoável.

Pode-se notar que dos 11 participantes ao menos 7 parcipantes classificaram que 
VIII Congresso Brasileiro de Informática na Educação (CBIE 2019)

Anais do XXV Workshop de Informática na Escola (WIE 2019)

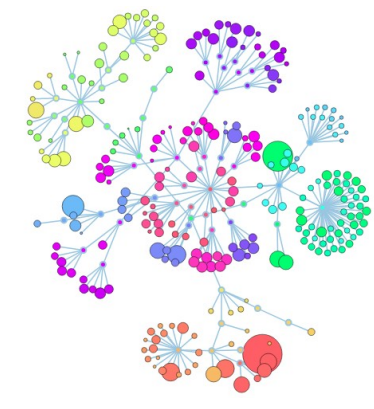

(a)

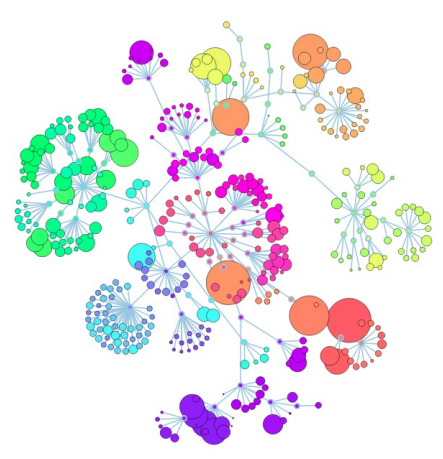

(c)

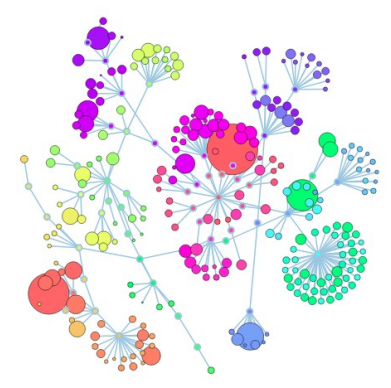

(b)

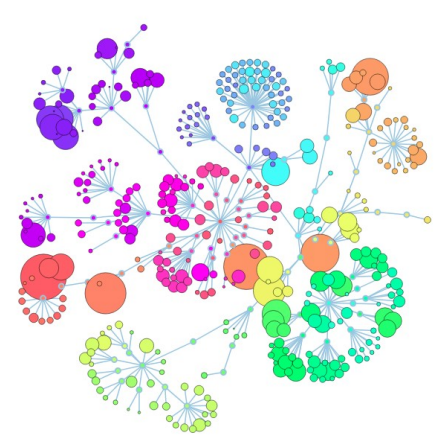

(d)

Figura 3. Evolução da ferramenta Gnosis ao longo das versões. As figuras (a), (b), (c) e (d) ilustram as versões $v 1, v 2, v 3$ e $v 4$ (vide Tabela 2) via métrca $K L o C$ para visualização de código. Nós maiores indicam maior acoplamento entre instrução por arquivo

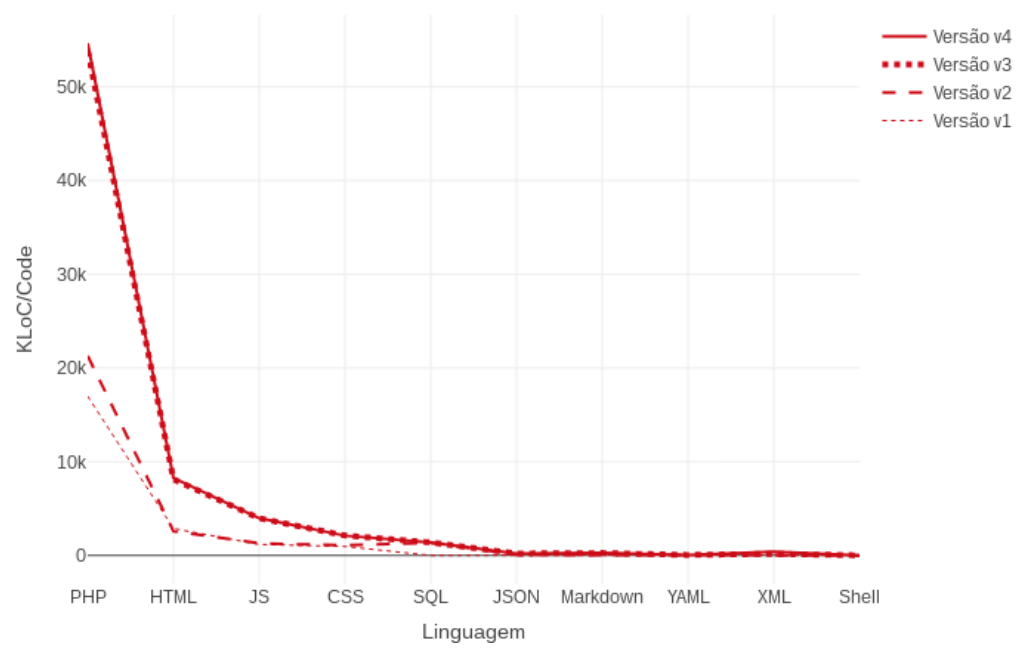

Figura 4. Variabilidade de Linguagens usadas vs. KLoC.

a ferramenta é excelente com respeito as questões $(Q 1-Q 6)$. Também, pode-se notar que em todas as questões $(Q 1-Q 6)$ nenhum participante considerou a ferramenta ruim sobre o ponto de vista do critério analisado. Observando-se as questões $(Q 4)$ e $(Q 5)$ 
VIII Congresso Brasileiro de Informática na Educação (CBIE 2019)

Anais do XXV Workshop de Informática na Escola (WIE 2019)
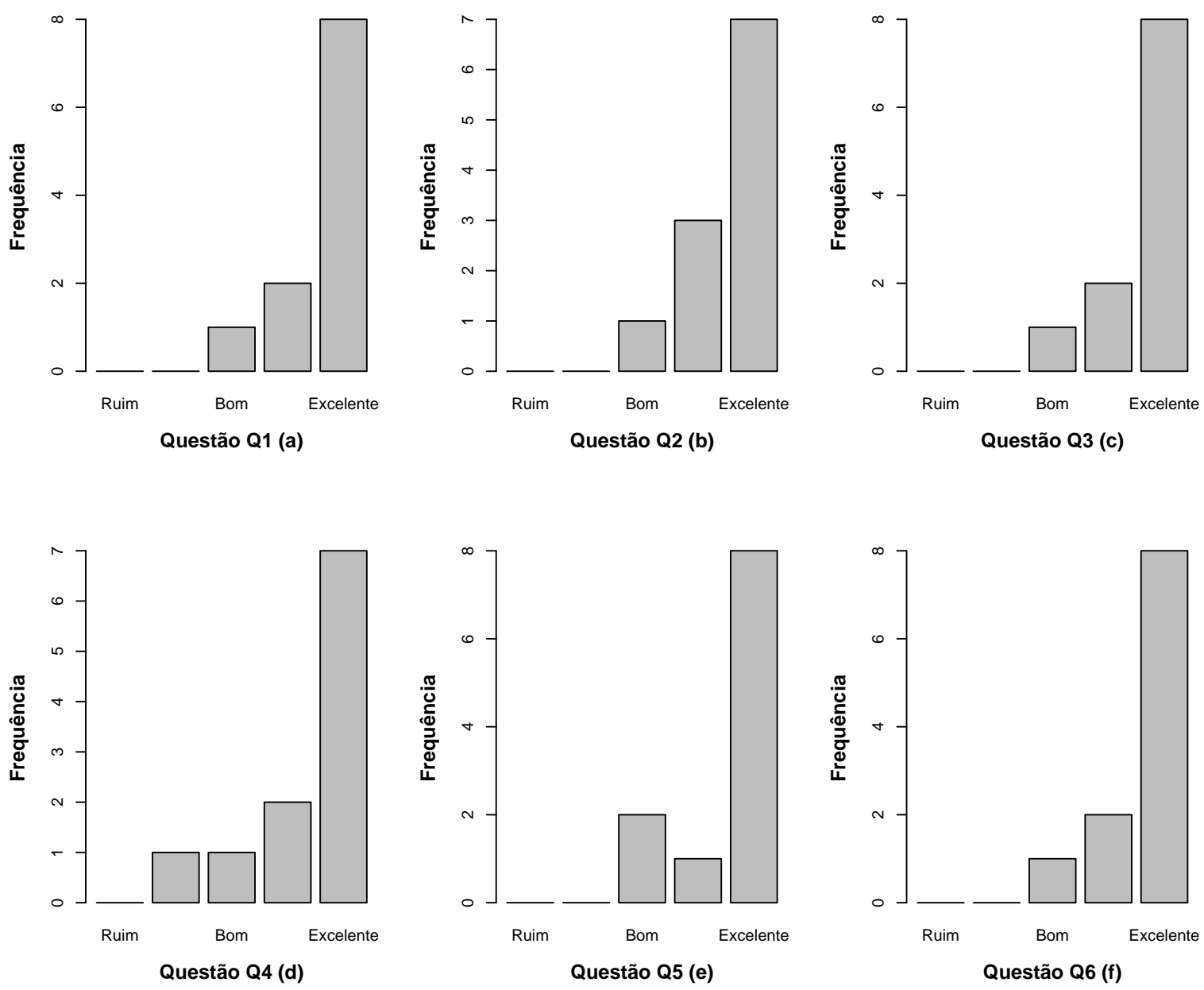

Atributos de Usuabilidade

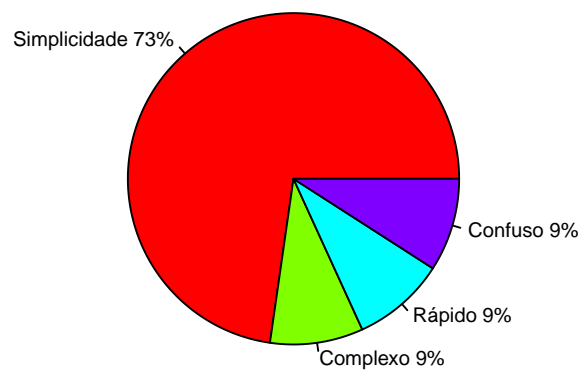

Questão Q7 (g)

Figura 5. A Figura 5 (a)-(f) ilustra as questões Q1-Q6 em gráficos de barra usando a escala no eixo-x com Ruim, Razoável, Bom, Muito Bom e Excelente versus a Frequência no eixo-y; A Figura 5 (g) destaca os atributos de qualidade avaliados pelos estudantes (Q7) 
VIII Congresso Brasileiro de Informática na Educação (CBIE 2019)

Anais do XXV Workshop de Informática na Escola (WIE 2019)

nota-se que ambas apresentam uma ligeira divergência em relação as outras questões pois foram potuados os itens como ruim e bom para as questões $(Q 4)$ e $(Q 5)$ respectivamente. Nesse caso, ambas questões indicam aspectos relacionados a usabilidade da ferramenta sobre padrões de cores, disposição dos menus, disposição dos botões e elementos visuais. A Figura 5 (g) nota-se que $73 \%$ dos participantes sugerem que a ferramenta possui o atributo simplicidade e menos de $27 \%$ dos participantes indicaram que a ferramenta é complexa, rápida ou confusa.

\begin{tabular}{c|c|c|c|c|c|c|c|c|c|c|c}
\hline Questão & \multicolumn{3}{|c}{ Ruim } & \multicolumn{2}{l}{ Razoável } & \multicolumn{2}{c}{ Bom } & Muito Bom & \multicolumn{2}{c}{ Excelente } \\
\hline \hline & $\#$ & $\%$ & $\#$ & $\%$ & $\#$ & $\%$ & $\#$ & $\%$ & $\#$ & $\%$ & $\sum \%$ \\
Q1 & - & - & - & - & 1 & 9,09 & 2 & 18,18 & 8 & 72,72 & $100 \%$ \\
Q2 & - & - & - & - & 1 & 9,09 & 3 & 27,27 & 7 & 63,63 & $100 \%$ \\
Q3 & - & - & - & - & 1 & 9,09 & 2 & 18,18 & 8 & 72,72 & $100 \%$ \\
Q4 & - & - & 1 & 9,09 & 1 & 9,09 & 2 & 18,18 & 7 & 63,63 & $100 \%$ \\
Q5 & - & - & - & - & 2 & 18,18 & 1 & 9,09 & 8 & 72,72 & $100 \%$ \\
Q6 & - & - & - & - & 1 & 9,09 & 2 & 18,18 & 8 & 72,72 & $100 \%$ \\
\hline $\bar{m}$ & - & - & 0,16 & 1,51 & 1,16 & 10,60 & 2,00 & 18,18 & 7,66 & 69,69 & $100 \%$ \\
\hline \hline
\end{tabular}

Tabela 3. Sumarização dos resultados encontrados

A Tabela 3 sintetiza os dados obtidos no estudo. Por se tratar de um estudo de viabilidade usando questionário e dada a importância em se assegurar a confiabilidade em relação as escalas definidas o teste de Cronbach alfa [Eunseong 2015] foi usado. O teste de Cronbach alfa é útil pois permite estabelecer a inter-relação entre as respostas obtidas em um questionário. Assim, aplicando-se o teste obtemos $\alpha=0,98$ no intervalo de confiança de $95 \%$ para as $k=6$ questões, o que sugere consistência interna do questionário em relação a inter-relação da escala definida para as questões $Q 1-Q 6$.

Em resumo, observando-se a coluna Excelente, nota-se a porcentagem média da pontuação dos participantes em relação as questões $(Q 1-Q 6)$. Dessa forma, considerando-se a hipótese inicial de pesquisa e os dados relativos a coluna - Criério de Excelente (vide Equação 3) - pode-se inferir que a hipótese de pesquisa $H_{a}$ não deve ser rejeita uma vez que na média $\approx 69,69 \%$, os participantes sugerem a existência de atributos de qualidade destinados a usabilidade.

$$
m_{Q 1-Q 6}=\frac{(4 * 72,72)+(2 * 63,63)}{6} \approx 69,69 \%
$$

Com relação à questão qualitativa $Q 7$ - por se tratar de uma questão aberta e descritiva - optamos por selecionar, uma das respostas e reproduzi-la abaixo:

Q7 Resposta - "Pensando no fator de usabilidade do site, eu pensaria na questão da responsividade do mesmo, quando mudado o meio de acesso, como por exemplo celulares, se ao usar o site nesses dispositivos o mesmo ainda manterá seu layout ou se adequará ao browser nestes dispositivos. Outra situação, seria a coloração que está "bem clara", e que isso possa gerar um certo incomodo no momento do uso. E, aumentar o campo do formulário resumo, para facilitar a visualização do texto, em toda sua dimensão.”. 
VIII Congresso Brasileiro de Informática na Educação (CBIE 2019)

Anais do XXV Workshop de Informática na Escola (WIE 2019)

A partir da reprodução do texto da resposta da questão $(Q 7)$, nota-se que o participante relata a existência de dois defeitos relacionados com a usabilidade da ferramenta. Um deles refere-se ao padrão de cores usado; e o segundo defeito, diz respeito ao tamanho do campo de submissão de resumos. Embora ambos os defeitos não sejam de alta severidade, a sua identificação é importante para se promover uma melhora no uso geral da ferramenta.

\section{Conclusão}

Neste trabalho foi apresentada uma ferramenta web (Gnosis) para apoiar o workflow de submissão de trabalhos acadêmicos. O trabalho relata, uma metodologia como diretriz para aplicação de um estudo de viabilidade para caracterizar atributos de qualidade da ferramenta proposta. Em especial, no estudo de viabilidade pode-se evidenciar ( $i$ ) a evolução da ferramenta desde 2016 - considerando quatro versões diferentes, número de linhas de código $(K L o C)$ e representação gráfica via visualização de código; e (ii) que do ponto de vista dos participantes do estudo de viabilidade, existem atributos de qualidade que permeam a ferramenta, sobretudo de usabilidade.

\section{Referências}

Antonio, E. A. (2019a). Github - paper-wei-2019. Disponível em: "https://github.com/ aceiro/paper-wei-2019". Acessado em set. de 2019.

Antonio, E. A. (2019b). Portal de eventos asser. Disponível em: "http://eventosasser.com. br". Acessado em set. de 2019.

Danial, A. (2019). Cloc: Count lines of code. Disponível em: "https://github.com/ AlDanial/cloc". Acessado em set. de 2019.

Eunseong, C. e Seonghoon, K. (2015). Cronbachs coefficient alpha: Well known but poorly understood. Organizational Research Methods, 18(2):207-230.

Gil, A. C. (2002). Como elaborar projetos de pesquisa. São Paulo: Atlas.

Joost, C. F. W. e Dimitra, D. (2010). Five-point likert items: t test versus mann-whitneywilcoxon. Practical Assessment, Research \& Evaluation.

MEC (2016). Resolução CNE/CES 5/2016. Brasília - DF.

Oliveira, E. S. et al. (2018). Identificação automática de estilos de aprendizagem: Uma revisão sistemática da literatura. In Anais do XXVI Workshop sobre Educao em Computao, Porto Alegre, RS, Brasil. SBC.

Pressman, R. (2009). Engenharia de Software - 7.ed. McGraw Hill Brasil.

Sauro, J. e Dumas, J. S. (2009). Comparison of three one-question, post-task usability questionnaires. In Proceedings of the SIGCHI Conference on Human Factors in Computing Systems, CHI '09, pages 1599-1608, New York, NY, USA. ACM.

Sullivan, G. M. e Artino, A. R. (2013). Analyzing and interpreting data from likerttype scales. Journal of graduate medical education.

Wohlin, C. (2012). Experimentation in Software Engineering. Springer Publishing Company, Incorporated.

Zaninotto, F. (2014). Codeflower source code visualization. Disponível em: "http://www. redotheweb.com/CodeFlower". Acessado em set. de 2019. 